\title{
The Performance of Polarization Switched-QPSK (PS-QPSK) in Dispersion Managed WDM Transmissions
}

\author{
P. Serena, A. Vannucci and A. Bononi \\ Università degli Studi di Parma, dip. Ingegneria dell'Informazione, v.le G. Usberti 181/A, 43124 Parma \\ (Italy),凶paolo.serena@unipr.it
}

\begin{abstract}
We investigate for the first time the performance of PS-QPSK in WDM nonlinear propagation. We show that the theoretical performance improvement of PS-QPSK compared to PDM-QPSK is even larger in nonlinear propagation.
\end{abstract}

\section{Introduction}

Along with the current renewed interest in coherent high-speed optical communications, polarization division multiplexed (PDM) modulation formats are attracting increasing attention, due to the possibility of exploiting the extra degrees of freedom provided by the signals state of polarization (SOP), to increase the bit-rate. In such a general framework, PDM- quadrature phase shift keying $(\mathrm{QPSK})^{1}$ is a simple instance of a broader class of simultaneous phase\&polarization shiftkeying formats, firstly analyzed in the first age of coherent optical communications in the early 90 's ${ }^{2}$. While PDM-QPSK is currently the most investigated among the constant-envelope formats, due to the little overhead in the transmitter (a simple polarization beam combiner that puts together two orthogonally polarized QPSK tributary subchannels) and to the good compromise between spectral and power efficiency, other solutions with attracting features are being proposed.

The polarization-switched-QPSK (PS-QPSK) format analyzed by Karlsson and Agrell $^{3}$ reaches an increased power-efficiency at the expense of a reduced bandwidth efficiency and a minimal complexity increase in the transmitter (two XOR logic gates). Such effects are achieved in PSQPSK by relying on a $2^{3}$-symbols constellation, where a polarization-switching bit further modulates a two-bit QPSK constellation. The polarization switching ensures a $\sqrt{2}$ increase in the minimal distance $d_{\min }$ between symbols (although the average distance is the same) compared to PDM-QPSK, hence a theoretical $1.76 \mathrm{~dB}$ asymptotic power gain, at the expense of a $4 / 3$ larger transmitted signal bandwidth (at equal bit-rate). $\mathrm{In}^{3}$, performance of single-channel PS-QPSK is evaluated in back-to-back transmission, i.e. in an additive-noise limited scenario.

In this work, we analyze by simulation the PSQPSK format in a realistic scenario. A wave-

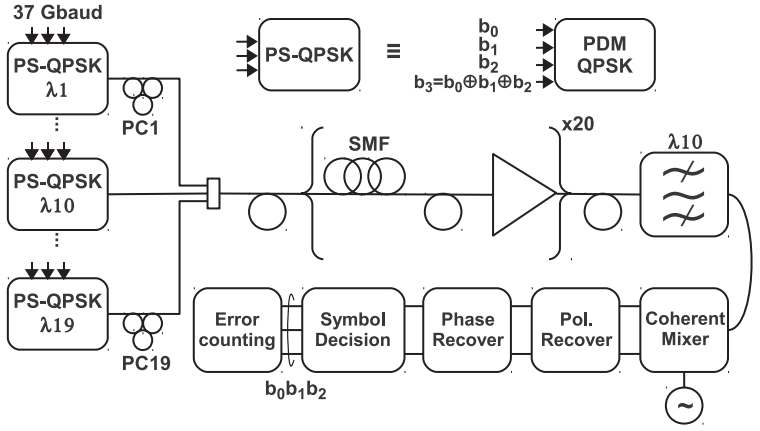

Fig. 1: PS-QPSK simulated setup.

length division multiplexing (WDM) field is sent over a multispan dispersion-managed fiber link, where nonlinear Kerr effect is present, as well as fiber birefringence. Although slowly adaptive polarization demultiplexing is performed in our simulations, the fast nonlinear polarization rotations (XPolM) induced by the Kerr effect, along with cross phase modulation (XPM), induce penalties at the receiver. We show that, for PS-QPSK, the beneficial effect of increased power-efficiency in the linear regime is paired with the beneficial effect of increased symbol rate in the nonlinear regime, i.e., provided that adjacent channel interference is not an issue with a $50 \mathrm{GHz}$ WDM grid, the larger bandwidth of each channel mitigates cross-channel nonlinear effect; this is possibly due to an averaging of the Kerr effect, produced by the increased dispersion.

\section{Numerical Setup}

We implemented PS-QPSK following the diagram of Fig. 1. The PS-QPSK transmitter was a standard PDM-QPSK transmitter using the coding rule of ${ }^{3}$, sketched in the top of Fig. 1 . In each transmitter the laser light was modulated with nested QPSK Mach-Zehnder modulators driven by 1024 symbols quaternary de Bruijn sequences at $R=28 \cdot \frac{4}{3} \simeq 37$ Gbaud, with non-return to zero shaped pulses. Before wavelength multiplexing, channels were filtered over a bandwidth 
of $50 \mathrm{GHz}$, and their SOP was randomly set through polarization controllers (PC). The channel spacing was $50 \mathrm{GHz}$, for a total of 19 channels. Before transmission we inserted a precompensating fiber of $-850 \mathrm{ps} / \mathrm{nm}$, while at the end of the link a post-compensating fiber set the overall cumulated dispersion to zero. The optical link had $20 \times 100 \mathrm{~km}$ spans of $D=17 \mathrm{ps} / \mathrm{nm} / \mathrm{km}$ fiber with nonlinear index $\gamma=1.3 \mathrm{~W}^{-1} \mathrm{~km}^{-1}$, dispersion managed with in-line residual dispersion of $50 \mathrm{ps} / \mathrm{nm} / \mathrm{span}$. Each fiber was simulated as the concatenation of 50 random birefringent waveplates without polarization mode dispersion (PMD). The optical propagation was modeled with the coupled nonlinear Schrödinger equation and solved by the split step Fourier algorithm using the open source platform Optilux ${ }^{4}$. We assumed noiseless propagation and added all the amplified spontaneous emission (ASE) noise at the end of the line, corresponding to a noise figure per lineamplifier of $6 \mathrm{~dB}$. The received signal, after passing through an optical filter of bandwidth $2 R$ and a polarization beam splitter, was combined with an ideal local oscillator in a $90^{\circ}$ hybrid. After the mixer, the four signals were filtered with bandwidth $0.7 R$, sampled at twice the symbol rate, and then digitally processed with: polarization recovery, Viterbi carrier phase estimation ${ }^{5}$ and symbol decision with minimum-distance detection ${ }^{6}$. The number of taps of the Viterbi estimators was optimized in each case.

The PDM-QPSK setup used the same blocks except for $R=28$ Gbaud, PDM-QPSK transmitters with all bits $b_{i}, i=0,1,2,3$ of Fig. 1 independently generated and a standard receiver where the polarization tributaries are detected individually ${ }^{1}$. Note that the bit rate is the same for the two formats (112 Gbit/s) since PDM-QPSK carries 4 bits/symbol while PS-QPSK only 3 bits/symbol.

The performance was measured in terms of the Q-factor extrapolated from the bit error rate (BER). The BER has been obtained with Monte Carlo simulations counting at least 100 errors. Finally, the BER was averaged over 10 different simulations with different random seeds. For a fair comparison, the same SOP and de Bruijn seeds were used for both PDM-QPSK and PS-QPSK.

\section{Results and Discussion}

The gain of PS-QPSK in back-to-back transmission w.r.t PDM-QPSK can be inferred from the simplified description of Fig. 2 (top row). We observe the phase-decision regions for both PDM-

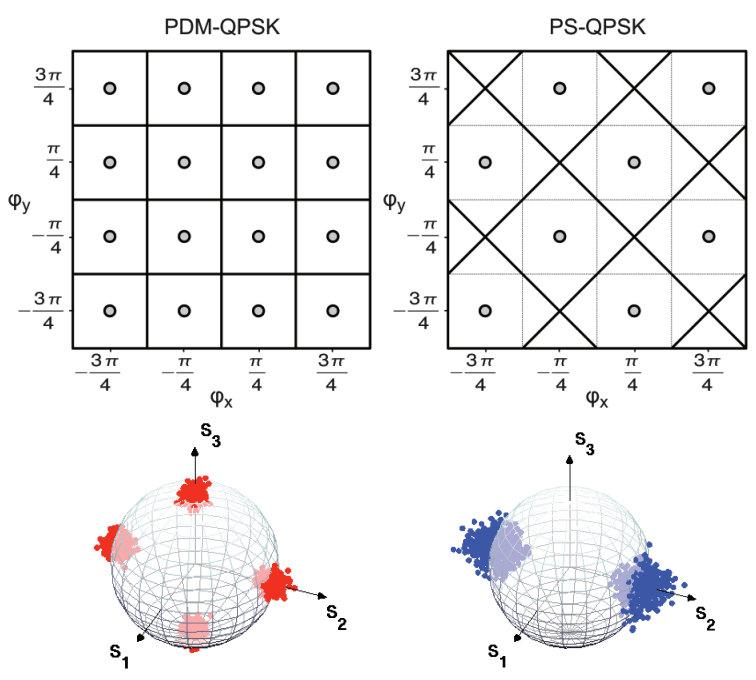

Fig. 2: Top-row: phase-decision regions for the two polarizations. Bottom-row: SOP over the Poincaré sphere. Left: PDM-QPSK. Right: PS-QPSK.

QPSK and PS-QPSK. Even if such description neglects the impact of the signal amplitude in the PS-QPSK correlation detection, it is of great help to understand how PS-QPSK makes an efficient use of the available phase-plane. From the figure it is clear the $\sqrt{2}$ distance gain of PS-QPSK w.r.t PDM-QPSK. Another difference between PDMQPSK and PS-QPSK can be searched over the Poincaré sphere. Here the special encoding rule of PS-QPSK (see Fig. 1(bottom)) makes its SOP switch between $\pm s_{2}$ axis, while PDM-QPSK can take four different SOP configurations. For this reason we expect different behaviors in the nonlinear regime due to a different XpolM effect.

We measured the average Q-factor of both formats working at $112 \mathrm{Gbit} / \mathrm{s}$ at different average power levels, whose results are depicted in Fig. 3. We observe a typical ascending region where performance is limited by the ASE noise (linear regime). For instance, we note that PS-QPSK shows a gain w.r.t PDM-QPSK of $0.45 \mathrm{~dB}$ at Qfactor of $8 \mathrm{~dB}$, close to the theoretical gain measured in ${ }^{3}(0.70 \mathrm{~dB} @ Q$-factor $8 \mathrm{~dB})$. The most interesting behavior is in the descending region of the $Q$-factor (nonlinear regime). Here the gain increased to $2.2 \mathrm{~dB}$ at the same Q-factor, making PS-QPSK largely more tolerant to nonlinear impairments than PDM-QPSK. Even if not reported here, we observed that the gain exists for any tested random seed.

To further investigate the nonlinear robustness of PS-QPSK we tested the link in single channel propagation. Fig. 4 depicts the corresponding Q-factor, showing that the nonlinear gain of PS- 


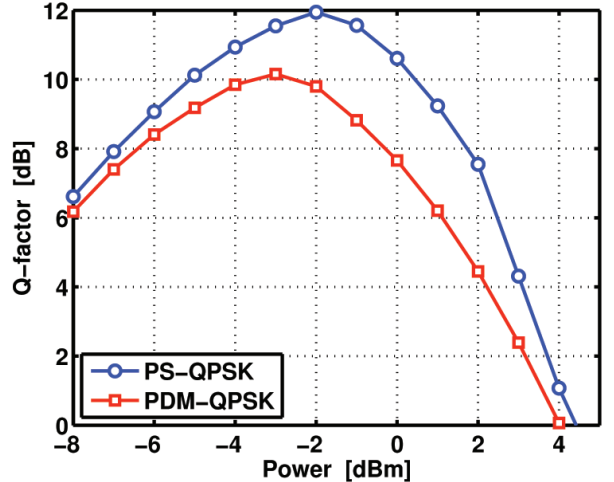

Fig. 3: 112 Gbit/s 19-channels PDM-QPSK and PS-QPSK performance.

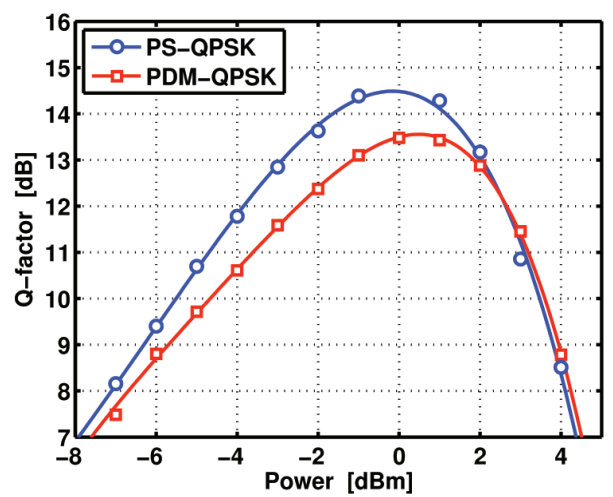

Fig. 4: Same as Fig. 3 but single channel propagation.

QPSK is strongly reduced compared to Fig. 3, an indicator that one of the reasons of the PS-QPSK robustness is a wide tolerance against XpolM.

To complete the picture, we tested PDM-QPSK at $R=37$ Gbaud to understand the role of the symbol rate on the performance. Following the same tests as before, we measured the average Q-factor shown in Fig. 5. Here we reported the same PDM-QPSK curve of Fig. 3 and the new one at 37 Gbaud. While in linear region we observe a natural penalty due to the increased bandwidth of the optical filter (scaled with $R$ ), in nonlinear region PDM-QPSK at 37 Gbaud recovers the gap, reaching the Q-factor of PDM-QPSK at $28 \mathrm{Gbaud}$. We thus conclude that increasing the symbol rate is a good way to mitigate nonlinear impairments, and hence PS-QPSK, being a 37 Gbaud format, makes an efficient use of such augmented symbol rate.

The final test concerns the impact of the Viterbi number of taps on PS-QPSK performance. Fig. 6 shows the average $Q$-factor vs. number of taps at different power levels. We note that large powers call for few taps, while the opposite occurs for small powers. This is in agreement with the ob-

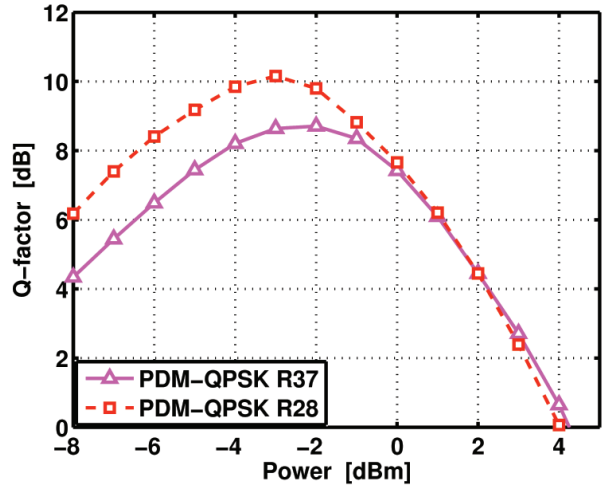

Fig. 5: PDM-QPSK at 28 and 37 Gbaud.

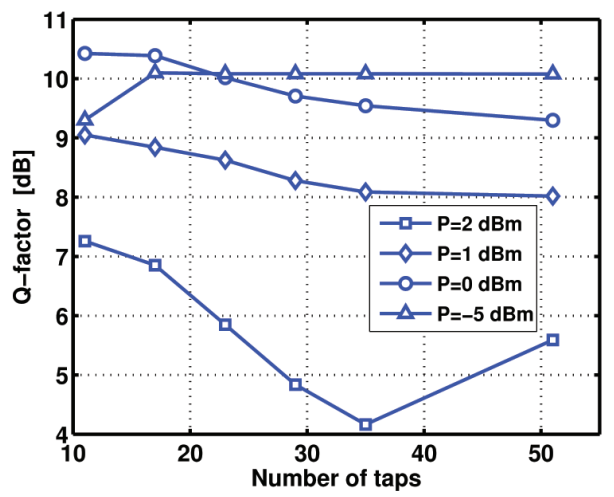

Fig. 6: Impact of Viterbi number of taps in PS-QPSK.

servations done for PDM-QPSK ${ }^{7}$, where in nonlinear regime a reduced number of taps allows to better estimate a phase shift induced by nonlinearities, which appear as a low-pass process.

\section{Conclusions}

We estimated for the first time to our knowledge the performance of PS-QPSK in a practical link with Kerr effect and WDM transmission. We showed that the PS-QPSK power-efficiency in linear regime is paired with a high tolerance against cross-channel effects in nonlinear regime.

\section{Acknowledgment}

The authors thank J. Renaudier of Alcatel-Lucent, Bell Labs, France for useful discussions.

\section{References}

1 G. Charlet, C. R. Physique 9, (2008)

2 S. Betti et al., J. Lightw. Technol. 9, 514 (1991).

3 M. Karlsson et al., Opt. Exp. 17, 10814 (2009).

4 P. Serena et al., "Optilux Toolbox", available at www.optilux.sourceforge.net

5 A. J. Viterbi et al., IEEE Trans. Inf. Theory 29, 543 (1983).

6 S. Benedetto et al., Principles of Digital Transmission, Kluwer (1999).

7 A. Bononi et al., J. Lightw. Technol. 27, 3974 (2009). 